\title{
Variant of a volume-of-fluid method for surface tension-dominant two-phase flows
}

\author{
G BISWAS \\ CSIR- Central Mechanical Engineering Research Institute, Durgapur 713209, India \\ Indian Institute of Technology Kanpur, Kanpur 208016, India \\ e-mail: gtm@iitk.ac.in
}

MS received 30 March 2012; revised 27 December 2013; accepted 28 December 2013

\begin{abstract}
The capabilities of the volume-of-fluid method for the calculation of surface tension-dominant two-phase flows are explained. The accurate calculation of the interface remains a problem for the volume-of-fluid method if the density ratios of the fluids in different phases are high. The simulations of bubble growth is performed in water at near critical pressure for different degrees of superheat using combined levelset and volume-of fluid (CLSVOF) method. The effect of superheat on the frequency of bubble formation was analyzed. A deviation from the periodic bubble release is observed in the case of superheat of $20 \mathrm{~K}$ in water. The vapor-jet-like columnar structure is observed. Effect of heat flux on the slender vapor column has also been explained.
\end{abstract}

Keywords. Volume of fluid method; level set method; bubble growth; film boiling.

The volume-of-fluid (Welch \& Wilson 2000) and the level-set (Osher \& Sethian 1998) approaches, are the major routes followed in the paradigm of fixed-grid methods. In the level-set method (Osher \& Sethian 1998) the interface is defined by a level-set function $\phi$. This function is initialized as a signed distance function from the interface, positive on one side and negative on the other side of the interface. The interface itself is represented by the zero level of $\phi$. Having computed the velocity field, the front evolves as a solution of a transport equation for $\phi$. This leads to interface smearing and difficulties in preserving the mass conservation. The levelset method has the inherent strength that $\phi$ varies smoothly across the interface, which leads to accurate calculation of the curvature and the interface normal vector. Owing to its simplicity, the level-set method has been used in solving a wide variety of problems (Sethian 1999; Sussman 2003).

In the volume-of-fluid (VOF) method (Welch \& Wilson 2000), the volume of each fluid is calculated in all cells containing portions of the interface. A volume fraction $\alpha$ is defined for one particular fluid inside a cell as its material volume divided by the total cell volume. Hence $\alpha$ is zero or unity in pure fluid (vapor or liquid) cells and has a value of $0<\alpha<1$ in two-phase cells. Volume-of-fluid algorithms consist of three major parts, the first part is, the 
interface reconstruction method, which finds an explicit description of the interface in each twophase cell based on volume fractions at this time step, the second part is advection algorithm, which calculates the distribution of $\alpha$ at the next time step by solving an advection equation using the reconstructed interface and the velocity field, and the final part consists of surface tension model, which takes account of surface tension effects at the interface. In this context, it is worth mentioning that Popinet \& Zaleski (1999) developed a novel front tracking method to compute surface tension dominated flows.

A significant development of the interface representation was achieved by Youngs (1982) by introducing the concept of piecewise-linear method (piecewise-linear interface calculation, PLIC). An improved version of phase interface representation (LVIRA) was devised by Puckett et al (1997) and implemented successfully by Welch \& Rachidi (2002) and Agarwal et al (2004) to predict bubble formation in film boiling. Tomar et al (2005) have extended the method as a variant of CLSVOF (Coupled level set and volume of fluid) method to simulate bubble growth and ebullition cycle in water and R-134a at new critical and far-from-critical pressures. Juric \& Tryggvason (1998) have provided novel procedures of computing boiling flows.

The present article is an attempt to consolidate the capabilities of recently published volumeof-fluid methods by our group (Agarwal et al (2004), Tomar et al (2005), Gerlach et al (2006), Tomar et al (2008), Chakraborty et al (2009) and Ray et al (2010, 2012)).

The mass, momentum and energy conservation equations for the incompressible Newtonian fluids for the liquid and vapor phases are given by

$$
\begin{gathered}
\frac{\partial U_{i}}{\partial x_{i}}=0 \\
\rho\left(\frac{\partial U_{j}}{\partial t}+\frac{\partial U_{i} U_{j}}{\partial x_{i}}\right)=-\frac{\partial p}{\partial x_{j}}+\frac{\partial}{\partial x_{i}}\left(\mu \frac{\partial U_{j}}{\partial x_{i}}\right) \\
\left(\frac{\partial\left(\rho c_{p} \theta\right)}{\partial t}+\frac{\partial\left(\rho c_{p} \theta U_{j}\right)}{\partial x_{j}}\right)=\frac{\partial}{\partial x_{j}}\left(k \frac{\partial \theta}{\partial x_{j}}\right)
\end{gathered}
$$

Here $U_{j}, p, c_{p}, \rho, \theta, \mu$ and $k$ are the fluid velocity, pressure, specific heat, density, temperature, viscosity and thermal conductivity, respectively.

A rectangular domian $5 \lambda_{B} \times \lambda_{B}$ has been chosen for the present investigation. Where $\lambda_{B}=2 \pi \sqrt{3 \sigma /\left(\rho_{l}-\rho_{g}\right) g}$ is the Taylor's most dangerous wave length. The simulations are two-dimensional. The boundary conditions are symmetry conditions at the left and right boundaries

$$
\text { at } x=0 \text { and } x=5 \lambda_{B}: u=0 ; \frac{\partial v}{\partial x}=0 ; \frac{\partial \theta}{\partial x}=0
$$

Constant wall temperature condition is used on the solid-fluid interface

$$
\text { at } y=0 \quad \theta=\theta_{\text {sup }}
$$

Outflow boundary conditions are used on the top surface of the domain

$$
\text { at } y=\lambda_{B} \quad \frac{\partial u}{\partial y}=\frac{\partial v}{\partial y}=\frac{\partial \theta}{\partial y}=0 ; P=P_{\circ}
$$

The outlet pressure is the saturation pressure less the hydrostatic pressure difference from the initial film level to the outlet. The boundary condition at the vapor liquid interface is of special 
concern in this study. In order to address this issue, a suitable interface tracking method has to be implemented.

The presence of two different phases of the fluid requires handling of the phase interface. We advect the interface using enhancement of VOF method of Hirt \& Nichols (1981) due to Youngs (1982). The method of Youngs is implemented at the end of a time cycle to calculate the new density field through complience of conservation of mass for each cell

$$
\frac{\partial}{\partial t} \int_{V_{c}} \rho d V+\int_{S_{c}} \rho \mathbf{v} \cdot \mathbf{n} d S=0,
$$

where $V_{c}$ is the cell volume and $S_{c}$ is the cell surface. The symbol $\mathbf{v}$ is used for the fluid velocity. Once, the new cell densities are found, the cell void fractions are calculated using

$$
\alpha=\frac{\rho-\rho_{g}}{\rho_{l}-\rho_{g}}
$$

Here $\rho_{l}$ and $\rho_{g}$ are the densities of the saturated liquid and saturated vapor, respectively. The implementation of the method of Youngs has been well documented in Welch \& Wilson (2000), Rudman (1997) and Rider \& Kothe (1998). The curvature of the iterface within each two phase cell is determined using the procedure described by Welch \& Rachidi (2002) based on LVIRA method of Puckett et al (1997). The method requires minimization of the function

$$
G_{i j}(\mathrm{n})=\sum_{k, l=-1}^{1}\left\{\alpha_{i+k, j+l}-\hat{\alpha}(\mathbf{n}, l)_{i+k, j+l}\right\}^{2} .
$$

Here $\alpha_{i, j}$ is the actual void fraction of the cell $(\mathrm{i}, \mathrm{j})$ and $\hat{\alpha}(n, l)_{i, j}$ is the trial function that maps the line with surface normal $\mathbf{n}$ and offset length $l$ into a volume fraction of the cell $(\mathrm{i}, \mathrm{j})$. This minimization of the function $G_{i j}(\mathbf{n})$ is a nonlinear problem requiring an initial estimate for $\mathbf{n}$. This initial $\mathbf{n}$ is the value determined by the modified VOF method of Youngs (1982) as

$$
\mathbf{n}=\frac{\nabla \alpha}{|\nabla \alpha|}
$$

The momentum equations are augmented using the continuum surface tension model of Brackbill et al (1992) in the following way

$$
\rho(\widetilde{\alpha})\left(\frac{\partial v}{\partial t}+\mathbf{v} \cdot \nabla \mathbf{v}\right)=-\nabla P+\rho(\widetilde{\alpha}) \mathbf{g}+\nabla \cdot\left[\mu(\widetilde{\alpha})\left(\nabla \mathbf{v}+(\nabla \mathbf{v})^{T}\right)\right]+\sigma \kappa \nabla \widetilde{\alpha}
$$

where $\widetilde{\alpha}$ is a smoothened void field and $\kappa$ is the curvature of the surface defined by smoothened void field. The surface tension force is applied to the transition region at the interface. The density and viscosity vary with the void fields as

$$
\begin{gathered}
\rho(\widetilde{\alpha})=\widetilde{\alpha} \rho_{l}+(1-\widetilde{\alpha}) \rho_{g} \\
\mu(\widetilde{\alpha})=\widetilde{\alpha} \mu_{l}+(1-\widetilde{\alpha}) \mu_{g} .
\end{gathered}
$$

For the interface cells, we use the augmented momentum Equation (8), the modified conservation of mass and the energy jump condition. The discontinuity of the velocity field, the velocity 
gradients, and the viscosity are treated by smoothening. A detailed formulation is available in Agarwal et al (2004).

Using the level-set formulation due to (Chang et al 1996) the momentum transport equation for incompressible two-phase flow becomes

$$
\rho(\phi)\left(\frac{\partial \mathbf{v}}{\partial t}+\mathbf{v} \cdot \nabla \mathbf{v}\right)=-\nabla P+\rho(\phi) g+\nabla \cdot\left[\mu(\phi)\left(\nabla \mathbf{v}+(\nabla \mathbf{v})^{T}\right)\right]+\sigma \kappa \nabla H \phi
$$

and the LS function advection equation is

$$
\frac{\partial \phi}{\partial t}+\mathbf{v} \cdot \nabla \phi=0
$$

The density and viscosity are derived from the level-set function as

$$
\begin{aligned}
& \rho(\phi)=\rho_{g}[1-H(\phi)]+\rho_{l} H(\phi) \\
& \mu(\phi)=\mu[1-H(\phi)]+\mu_{l} H(\phi),
\end{aligned}
$$

where $H(\phi)$ is the Heaviside function,

$$
H(\phi)= \begin{cases}0 & \text { if } \phi \leq-\epsilon \\ \frac{1}{2}\left[1+\frac{\phi}{\epsilon}+\frac{1}{\pi} \sin \left(\frac{\pi \phi}{\epsilon}\right)\right] & \text { if } \phi \leq \epsilon \\ 1 & \text { if } \phi>\epsilon\end{cases}
$$

The local mean curvature is given by $\kappa=-\nabla . \mathbf{n}$

When discretizing the LS advection equation (12), the volume-of-fluid function $\alpha$ is also simultaneously solved from the following equation:

$$
\frac{\partial \alpha}{\partial t}+\nabla \cdot(v \alpha)=0
$$

Figure 1 (a) shows the periodic bubble release patterns with growing interface and varying vapor volume. The periodic growth and release of vapor bubbles is usually called ebullition cycle. Starting with a thin vapor layer, gradually the bubbles grow in size. Finally, the bubbles reach a limit at which they detach. The detached bubbles move upwards and leave the domain of interest. The released bubbles leave a very thin vapor film near the nodal position on the wall. Due to the vapor production at the interface the thin vapor layer again grows in size. The next bubbles tend to grow at the antinodes according to the Taylor's wave length $\lambda_{B}$.

The mechanism for the repeating bubble detachment pattern (not shown here) can be explained in the following way. After the bubbles are released from the surface, the vapor packets left behind experience a downward force due to surface tension. Subsequently the vapor packets are pushed down towards the film. The vapor packets impinge on the horizontal surface. The surface tension induced flow promotes the movement of pressure gradient driven impinging vapor packet towards the antinodes. The vapor turns upward to initiate an identical bubble release cycle. Similar patterns of bubble release were observed by Son \& Dhir $(1997,1998)$ in their axisymmetric simulations of saturated film boiling.

CLSVOF based Simulations were performed with a superheat of $20^{\circ} \mathrm{C}$ and a notable change in bubble dynamics took place. For a superheat of $10^{\circ} \mathrm{C}$, the bubble detachment took place alternatively from node and antinode. But for $20^{\circ} \mathrm{C}$ superheat, a very tall and slender vapor column formation was observed as shown in figure 1 (b). Similar profiles were observed in 

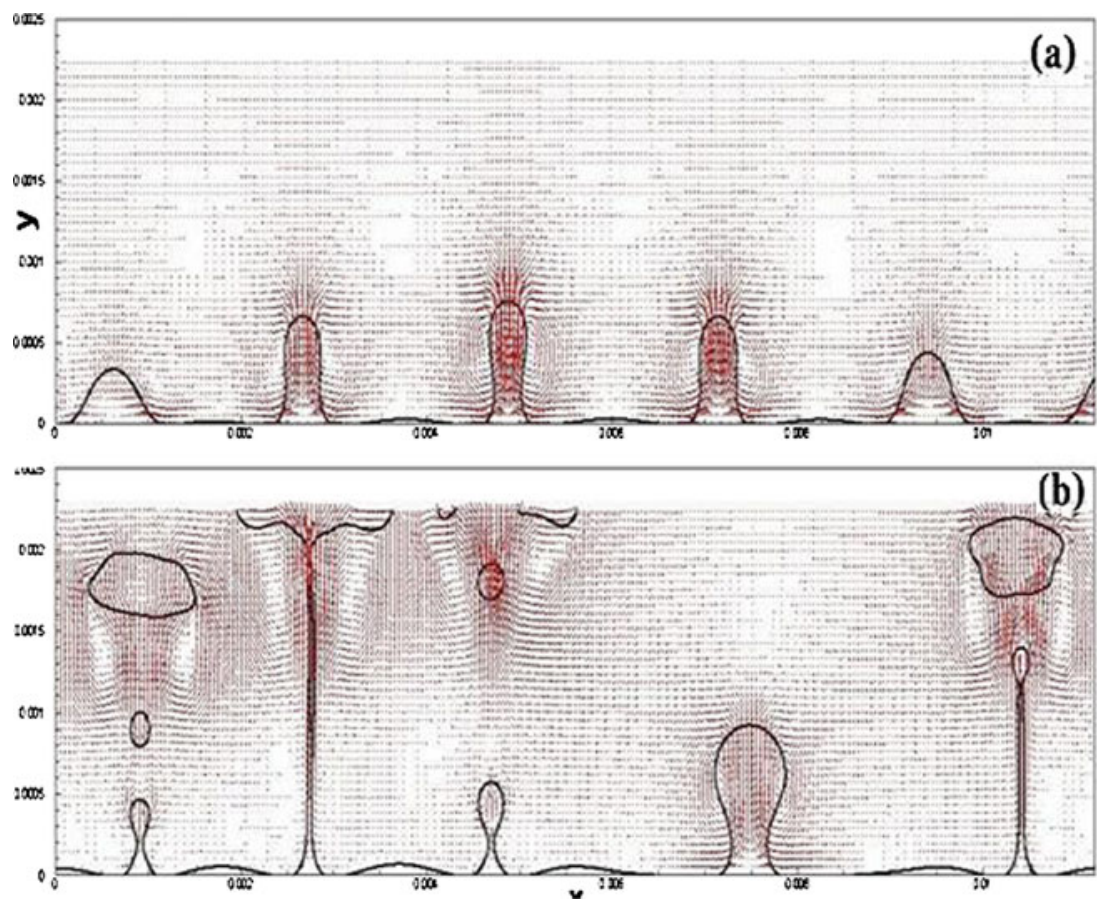

Figure 1. Interface profile with velocity vectors at a surface superheat of (a) $\Delta \mathrm{T}=10 \mathrm{~K}$, (b) $\Delta \mathrm{T}=20 \mathrm{~K}$.

experiments of Reimann \& Grigull (1975) when higher values of heat flux beyond a critical value were used. It was observed that after a critical value of heat flux, this change in bubble dynamics took place.

\section{Nomenclature}

$c_{p}, c_{v}$ specific heat at constant pressure/volume

Gr Grashof number

$g \quad$ gravitational acceleration

$H \quad$ height of computational domain

$h_{l g} \quad$ latent heat of vaporization

$k \quad$ thermal conductivity

$l \quad$ normal distance from cell center to phase interface

$n \quad$ interface normal vector, pointing into liquid phase

$P_{o} \quad$ saturation pressure in excess of hydrostatic pressure

$p \quad$ total pressure

$q \quad$ heat flux vector

$S_{c} \quad$ computational cell boundary surface

$S_{I} \quad$ phase interface surface

$t$ time

$u \quad \mathrm{x}$ component of velocity 
$U_{i}, U_{j}$ velocity vectors

$V \quad$ volume

$V_{c} \quad$ cell volume

$v \quad$ y component of velocity

$v \quad$ fluid velocity

$x \quad$ spatial coordinate in the horizontal direction

$x_{i}, x_{j} \quad$ space vectors

$y \quad$ spatial coordinate in the vertical direction

\section{References}

Agarwal D K, Welch S W J, Biswas G and Durst F 2004 Planer simulation of bubble growth in film boiling in near-critical water using a variant of the VOF method. J. Heat Transfer (ASME) 126: 329-338

Brackbill J U, Kothe D B and Zemach C 1992 A continuum method for modeling surface tension. $J$. Comput. Phys. 100: 335-354

Chakraborty I, Ray B, Biswas G, Durst F, Sharma A and Ghoshdastidar P S 2009 Computational investigation on bubble detachement from submerged orfice in quiescent liquid under normal and reduced gravity. Phys. Fluids 21: 062103-1-062103-17

Chang Y C, Hou T Y, Merriman B and Osher S 1996 A level set formulation of eulerian interface capturing methods for incompressible fluid flows. J. Comput. Phys. 124: 449-464

Gerlach D, Tomar G, Biswas G and Durst F 2006 Comparison of volume-of-fluid methods for surface tension-dominant two-phase flows. Int. J. Heat Mass Transfer 49: 740-754

Hirt C W and Nichols B D 1981 Volume of fluid (VOF) method for the dynamics of free boundary. $J$. Comput. Phys. 39: 201-225

Juric D and Tryggvason G 1998 Computations of boiling flows. Int. J. Multiphase Flow 24: 387-410

Osher S and Sethian J A Fronts propagating with curvature-dependent speed: Algorithms based on Hamilton-Jacobi Formulations. J. Comput. Phys. 79(1): 12-49

Popinet S and Zaleski S 1999 A front-tracking algorithm for accurate representation of surface tension. Int. J. Numer. Methods Fluids 30: 775-793

Puckett E G, Almgren A S, Bell J B, Marcus D L and Rider W J 1997 A high-order projection method for tracking fluid interfaces in variable density incompressible flows. J. Comput. Phys. 130: 269-282

Ray B, Biswas G and Sharma A 2010 Generation of secondary droplets in coalescence of a drop at a liquid-liquid interface. J. Fluid Mech. 655: 72-104

Ray B, Biswas G and Sharma A 2012 Bubble pinch-off and scaling during liquid drop impact on liquid pool. Phys. Fluids 24: 082108-1-082108-11

Reimann M and Grigull U 1975 Warmeubergang bei freier Konvektion und Filmsieden im kritischen Gebiet von Wasser und Kohlendioxid. Warme-und Stoffubertragung 8: 229-239

Rider W J and Kothe D B 1998 Reconstructing volume tracking. J. Comput. Phys. 141: 112-152

Rudman M 1997 Volume-tracking methods for interfacial flow calculations. Int. J. Numer. Methods Fluids 24: 671-691

Sethian J A 1999 Level set methods and fast marching methods. UK: Cambridge University Press

Son G and Dhir V K 1997 Numerical simulation of saturated film boiling on a horizontal surface. J. Heat Transfer (ASME) 119: 525-533

Son G and Dhir V K 1998 Numerical simulation of film boiling near critical pressure with a level set method. J. Heat Transfer (ASME) 120: 183-192

Sussman M 2003 A second order coupled level-set and volume-of-fluid method for computing growth and collapse of vapor bubbles. J. Comput. Phys. 180: 110-136

Tomar G, Biswas G, Sharma A and Agrawal A 2005 Numerical simulation of bubble growth in film boiling using a coupled level set and volume-of-fluid method. Phys. Fluids 17: 112103-1-112103-13 
Tomar G, Biswas G, Sharma A and Welch S W J 2008 Multimode analysis of bubble growth in saturated film boiling. Phys. Fluids 20: 092101-1-092101-7

Welch S W J and Wilson J 2000 A volume of fluid based method for fluid flows with phase change. $J$. Comput. Phys. 160: 662-682

Welch S W J and Rachidi T 2002 Numerical simulation of film boiling including conjugate eat transfer. Numer. Heat Transfer B 42: 35-53

Youngs D L 1982 Time-dependent multi-material flow with large fluid distortion. In: K W Morton and M J Baines (eds), Numerical methods for fluid dynamics. New York: Academic Press 